\title{
Clomiphen-Zitrat in der Behandlung anovulatorischer
} Blutungen

\begin{tabular}{|l|l|l|}
\hline R.A. & \\
\hline R.B. & Puebla \\
\hline
\end{tabular}

Medical College of Georgia, Augusta, USA

Starke Blutungen bei anovulatorischen Zyklen werden insbe-sondere zu Beginn und gegen das Ende der reproduktiven Phase der Frau beobachtet. Da diese Störung primär im Ausfall der Ovulation liegt, sollte die Behandlung diese pathogenetische Ursache korri-gieren. Die Verfasser untersuchten deshalb die Wirkung des auf dem Wege der Gonadotropinauslösung zur Ovulation führenden Clomiphen auf die anovulatorischen Blutungen.

Es wurden 26 Patientinnen im Alter von 12 bis 35 Jahren be-handelt. 24 von ihnen hatten seit der Menarche unregelmäßige Menstruationen, 15 hatten bereits vor Beginn der Behandlung eine oder mehrere Hormonkuren, in 23 Fallen wurden bereits Curetta-gen zur Behandlung vorgenommen.

Zu Beginn dieser Behandlung wurde in 20 Fallen eine Strich-curettage vorgenommen, welche in jedem Fall ein hyperplastisches oder zumindest proliferatives Endometrium ergab. Bei 6 weiteren mußte man sich mit vaginalen zytologischen Abstrichen begnügen (Virgo!), welche ebenfalls für anovulatorische Blutungen sprachen. Zu Beginn der Serie wurde das Clomiphen in der Dosierung von 25 bis 200 mg/die über 20 Tage oder als Dauertherapie gegeben, mit Zunahme der Erfahrung reduzierte sich die Dosis auf 25 bis 75 mg/die während 5-10 Tagen. Das Eintreten von ovulatorischen Zyklen wurde durch die Basaltemperatur, die Histologie des Endometriums, Untersuchung des Zervikalschleims, vaginale Zytologie, Pregnandiolausscheidung im Urin oder dem Eintreten einer Schwangerschaft festgestellt.

Ergebnisse: Bei 7 Patientinnen, welche Clomiphen-Zitrat allein oder, in einem Fall, kombiniert mit einem Nortestosteron-Präparat, während der Blutungsphase erhielten, stoppte die Blu-tung spätestens am 10. Tag, und es traten dann in 6 Fallen mit Sicherheit ovulatorische Zyklen auf. Gesamthaft ließen sich bei 24 der 26 Patientinnen ovulatorische Zyklen auslösen, wobei von total

* Originaltitel: Glomiphene citrate in the menagement of anovulatory uterine bleeding. Puebla und Greenblatt

155

129 Zyklen 105, d.h. 81,3\%, ovulatorisch waren. Nach Beendigung der Behandlung hatten 38,4\% weiterhin ovulatorische Zyklen. 16 der 26 Patientinnen waren verheiratet, bei zweien kam es unter der Behandlung zur Schwangerschaft. Als Mebenwirkungen stellten sich in 4 Fallen tastbare, zystische Ovarien, in 2 Fallen Wallungen ein.

Auf Grund dieser vorliegenden Ergebnisse glauben die Auto-ren, daß die ovulationsauslösende Wirkung von Clomiphen in der Behandlung anovulatorischer Blutungen eine Bereicherung des Arzneischatzes auf diesem Gebiet darstellt. 
W. Obolensky

Kommentar siehe S. 160

Aus: Amer.J. Obstet. Gynec. 90: 355-363 (1964); cit. Gynäk. Rdsch. 2: 155-156 (1965)

Die Anwendung und wahrscheinliche Wirkung von Clomiphen-Zitrat *

M. J. Whitelaw, L. R. Grams und W. J. Stamm

O’Connor Hospital, San José, California

Auch heute, nachdem die gonadotropen Hormone in größe-rem Ausmaße gebraucht worden sind, ist ihre Verwendung nicht einfach, weil sie 1. sehr schwer erhältlich sind, 2. zu

vergrößerten polyzystischen Ovarien und 3. zu Mehrlingsschwangerschaften führen und 4. ihre Wirkung nur so lange ausüben, als sie verab-reicht werden. Deshalb ergaben sich durch das synthetische Clomi-phenzitrat völlig neue Möglichkeiten in der gynäkologischen Endokrinologie, insbesondere bei der Ovulationsauslösung. Der vor-liegende Bericht stützt sich auf Beobachtungen bei 70 Patientinnen, von denen 55 verheiratet waren und wegen Sterilität behandelt wurden. Die Verteilung des Patientengutes und der Behandlungs-erfolg sind in Tabelle I wiedergegeben.

Die sogenannten Normalfälle waren solche

bei denen keine Ursache für die Sterilität gefunden werden konnte

und die reguläre

* Originaltitel: Clomiphene citrate: Its uses and observations on its probable action. 